\section{(B) luseriations}

\author{
oN
}

\section{THE NATURE - AND SYMPTOMS OF CARDIAC INFECTION IN CHILDHOOD. BY}

F. J. POYNTON, M.D., F.R.C.P.LoND.,

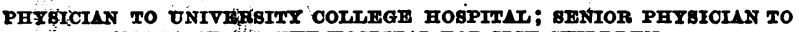
OUT-PATIEN

\section{II.-RHEUMATIC HEART DISEASE.*}

First Attacks of Rheumatism in Childhood. Trese bring as to the threshold of the early development of organic disease. Among such cases we find all grades of severity, from those-fortunately rare-which prove rapidly fatal, and in which the infective element reaches its acme of intensity, to those which are so transient and vague that we are left doubtful of their significance. From $a$ situdy of the latter we realize that a considerable number elode discovery by parents and doctors, and that as a resilt the early detection of cardiac affections can be.but a relative accomplishment, the accuracy of which will only be tncreased by continual improvement in the education of the public and in our clinical methods.

It is here that a general idea of the relative frequency of the well recognized manifestations of the disease beedmes of some practical importance, and the following figures, based upon 524 examples which I believe to be first attacks, may be of service. These figures, however, can only be of relative value, for the proof that an attack was actually the first is often difficult.

When these children (all under twelve years of age) were first seen at the hospital the following conditions were present:

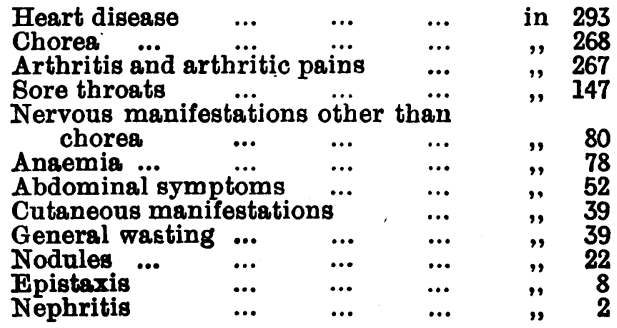

Two important groups may be recognized:

1. Cases with sore throat, arthritis, and morbus cordis.

2. Cases with chorea and morbus cordis.

For reasons that I have given in Researches upon Rheumatism I have included all cases of chorea as rheumatic in origin.

The sex incidence was as follows :

$$
\begin{array}{lllllll}
\text { Females } & \ldots & \ldots & \ldots & \ldots & 346 \\
\text { Males } & \ldots & \ldots & \ldots & \ldots & 169
\end{array}
$$

In 9 the sex was overlooked.

The percentages in this series were approximately

$$
\begin{array}{llllll}
\text { Females } & \ldots & \ldots & \ldots & \ldots & 69 \\
\text { Males ... } & \ldots & \ldots & \ldots & \ldots & 33
\end{array}
$$$$
\text { As nearly as possible } 2 \text { to } 1 .
$$

Age incidence in 364 cases:

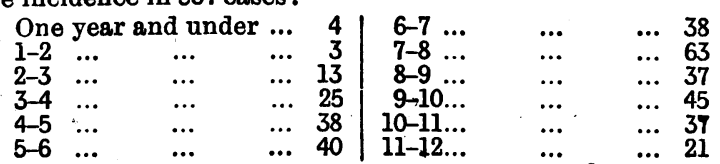

\section{Classification of the Chief Lesions of Rheumatio}

$$
\text { Heart Disease. }
$$

I think the clearest and most instructive method of approaching the subject is to commence with the earliest and often the least severe cardiac lesion-acute dilatation. This is an essential feature in the history of rheumatic cardiac affections, and must be clearly recognized. Then will come for description the most severe and most characteristic event-an acutely fatal carditis in a first attack. From these latter cases we learn the true

\footnotetext{
* The first article was printed in the JouRN 1 L of March 2nd, 1918.
}

meaning of a cardiac infection. Next will follow the three cardinal lesions of the textbooks-pericarditis, endocarditis, and myocarditis-which lead to the consideration of recurrent carditis and the final stages of infective carditis.

Whatever classification is adopted, the element of carditis will be found frequently complicating the individual lesions, but I shall endeavour to avoid a troublesome repetition of symptoms and consider the subject only with the view of illustrating special features of the rheumatic infection.

\section{ACUTE DILATATION.}

There are all grades in the: severity of acute dilatation. In very rare cases it may prove fatal without endocardial or pericardial involvement. Far more frequently it occurs with some mitral endocarditis. Again, it is the chief cause of cardiac failare in recurrent attacks of carditis. Lastly, there is a large group in which the dilatation either is not severe and may be recovered from rapidly, or may be slow in course and intractable.

We are concerned here with the mild acute cases recovering rapidly. The more severe and the chronic cases will be considered later

When it is recognized that some degree of dilatation may be the only cardiac lesion, and also that it complicates all the more severe rheumatic cardiac injuries, it will : be evident that this is not only the earliest clinical evidence we possess of rbeumatic heart disease, but also the most frequent.

I adrocate the value of making careful outlines of the deep cardiac dullness in this lesion and of tracing these outlines on to paper for future reference. Each outline should have fixed points, such as the left nipple and midsternum, marked on it, and should be completed with the date and a brief synopsis of the symptoms and signs. Though I am no believer in a rigid dependence upon cardiac percussion it is remarkable how accurate the results become by persevering with this rather laborious method. From such an experience we learn not only to appreciate the reality of simple dilatation but also to detect the part it takes in acute pericarditis, and in the exacerbations of rheumatism in hearts already injured by the disease. Another point we learn is the remarkable rapidity with which dilatation sometimes occurs even when a child has been in bed for some days. Without special care this may be easily overlooked, but it is quite in accord with other events that may happen to rheumatic child while resting in hospital. For example, a sore throat may suddenly develop, or chorea commence, or an arthritis show itself. These outbursts of infection constitute one of the peculiarities of the disease, and they suggest the sudden influx of a fresh wave of infection after the earlier one has been dealt with by the tissue cells.

\section{The Signs and Symptoms of Acute Dilatation of} the Heart of Mild Degree.

The ohief signs of acute dilatation of mild degree are not.difficult to ascertain or to remember when once they have been thoroughly studied, and may be summarized as follows:

If the child is leading its usual life there will be found shortness̃ of breath, pallor, and palpitation, and there may even be a fainting attack. The pulse is quickened, and easily com pressible.

The impulse of the heart is enfeebled and the cardiac dullness reaches to or beyond the left nipple line, and may be increase to the right. At the impulse the first sound is shortened, and at the pulmonary base the second sound is accentuated and may be reduplicated. Over the mitral area a soft systolic bruit mai be audible, which is often at its maximum just internal to the impulse, and is not conducted plainly toward the left axilla. There will probably be a slight rise in the temperature.

From the above description it is apparent that it may be far from easy to decide whether in any given case there is together with the dilatation some endocarditis of the mitral valve. A question I have had put to me is how to distinguish these two lesions, "and the answer, though not difficult to give, is necessarily incomplete.

If there is mitral endocarditis of some severity the apical murmur is conducted towards the axills and even if not nermanent will not disappear for many months, and long after the soft murmur of a dilatation will have vainished. Further, there is usually evidence of hypertrophy of the left ventricle supervening upon the dilatation. If the endocarditis is slight 
the murmur will nevertheless persist after all evidence of dilatation has disappeared. There remain a certain number of cases in which the dilatation is slow in recovery, and also some others in which in all probability a valvular murmur disappears with unusual rapidity, and then we may be in doubt. The uncertainty in the latter case is not in reality of practical importance, for if the murmur disappears and the heart becomes sound we can but be satisfied. If, however, the dilatation is more stubborn than usual there will be the signs of myocardial feebleness, and for a considerable time we must remain in doubt as to whether or not an endocarditis is responsible for this delay.

It is clear, then, that a satisfactory answer to the question sometimes requires time and repeated observation before it can be given.

There is no doubt, I think, that the rapid development of acute dilatation accounts for some of the cases in childhood in which, after a slight arthritis, the patient has been allowed to return quickly to ordinary life, and has then become suddenly short of breath or has collapsed with a syncopal attack. A thorough understanding of this possibility will serve as an added warning to watch carefully the early days of what may seem a very mild attack of rheumatism.

\section{FATAL FIRST ATTACKS OF CARDITIS.}

From the least severe of the rheumatic cardiac lesionsacute dilatation of slight degree-I pass now to the most severe, which brings into prominence the part taken by infective processes in human heart disease, and illustrates how completely they may destroy all attempts at recovery of the mechanical balance of the circulation. In such cases we must expect to find symptoms of great severity and of an unusual nature-for example, shivering attacks, vomiting, and diarrhoea, great muscular prostration and extreme illness; and we are also impressed by the rapid progress made by the cardiac disease. Thus pain over the precordium will be swiftly followed by severe dilatation and cardiac excitement, pericarditis will rapidly become general, and endocarditis appear. In a week or two the lieart is irreparably damaged. It is not, however, the pericarditis or valvular disease that alarms us most, but the general cardiac infection and the failure of the general vitality from the virulence of the disease.

These fatal first attacks are rare-and they are more often met with in young children or in those with a strong hereditary tendency to rheumatism.

I would draw particular attention here to the answer that these severe cases gives to those who hold that rheumatism is an attenuated pyaemia. A study of them must, I think, convince us that the illness is of a deadly virulence, and yet, as the necropsies prove, never approaches to the character of a pyaemia. The poisons may be chemically allied to those of pyaemia, but they certainly are as special in their action as the poisons of any other specific disease.

The fifteen cases upon which these observations are chiefly based fall into two main groups:

(a) Fulminating cases of acute carditis.

(b) Insidious cases in fragile children of feeble constitution.

In this latter group the disease is deceptively quiet in its onset, but none the less deadly. The four following examples well illustrate these fatal forms of carditis-the first three the fulminating group, the last the asthenic.

$$
\text { Example } 1 .
$$

A boy under 5 years of age, with a rheumatic history on the father's side, was seized with general illness a week before admission to hospital. At the outset he was prostrated with vomiting and diarrhoea and pains all over the body, and on admission he looked very pale and ill. 'I'here was arthritis of both knees, and there was great dilatation of the heart with a rapid pulse (140 per minute), and a systolic mitral murmur. Two days later general pericarditis was discovered, and bronchopueumonia developed. The temperature ranged between $103^{\circ}$ pueumonia developed. The temperature ranged between $103^{\circ}$ and $100^{\circ} \mathrm{F}$. The course was rapidly downhill, the liver became running pulse, with some albumin in a scanty urine, followed, running pulse, with some albumin in a scanty ur
and death occurred fourteen days after admission.

The necropsy showed recent pericarditis, great dilatation, The necropsy showed recent pericarditis, great dilatation,
and early mitral endocarditis. The diplococcus was isolated and early mitral endocarditis. The diplococcus was isolated
from the pericardial, pleural, and arthritic exudations, and from the pericardial, pleural,

\section{Example 2.}

A girl, aged 33 years, was admitted to hospital very ill and with signs of severe cardiac disease. Her father had suffered from rheumatic fever and a sister had died of the same disease. Four weeks before admission, after developing a sore throat she became generally ill, with pains all over the body and swelling of the hands and ankles.

In hospital sour perspirations were noticed with high fever ; dilatation, general pericarditis, and mitral endocarditis followed. Later nodules appeared together with the usual signs of cardiac failure. Finally all food was refused, and the child died after lingering for five weeks.

Example 3.

A boy, aged $7 \frac{1}{2}$ years, had suffered ten days before admission with general malaise, diarrhoea, sleeplessness, and cough with general malaise, diarrhoea, sleeplessness, and cough, rapidly followed by arthritis of the ankles, fingers, toe-joints, days later he was allowed to get up. The same night he became days later he was allowed to get up. The same night he became
deirious. When admitted to hospital there was severe carditis deilirious. When admitted to hospital

The necropsy showed a large pericardial effusion with simple aortic and malignant mitral endocarditis. Reference will be made later to this most remarkable case.

The next example illustrates the asthenic form of fatal carditis :

\section{Example 4.}

A girl, aged $4 \frac{1}{2}$ years, who was pale, fat, and flabby, had suffered for some weeks before admission from indefinite $i ! 1$ ness. The chief early symptoms were muscular feebleness and general prostration, with progressive pallor and loss of appetite. Then there developed a slight but definite chorea. When admitted the child was very ill and the pulse rapid (130), the heart was greatly dilated and the sounds faint with a soft systolic apical murmur. The next day general pericardial friction became audible. The temperature ranged around $100^{\circ} \mathrm{F}$., and the extremities were cold. There was no sign of rallying to treatment and four days later she died from cardiac failure, the temperature rising in the last twenty-four hours to $104^{\circ} \mathrm{F}$.

The necropsy showed recent universal pericardial adhesions, great dilatation, mitral and tricuspid endocarditis, plastio preat dilatation, mitral and tricuspid endocarditis, plastic pleurisy on both sides and
left internal jugular vein.

These cases give a general indication of the clinical course of this most fatal form of rheumatic heart disease. We do not see cyanosis and distended jugular veins There is no pronounced development of oedema with great enlargement of the liver. The child is more often ashen pale, the liver palpable, and the oedema a mere puffiness of feet and ankles. Death is often sudden from a terminal syncope, and the general appearance one of a severe toxaemia with a high degree of oxygen want.

In cases such as these Dr. Paine and I have demonstrated and isolated the strepto-diplococcus, a result only to be expected from the experience of bacteriologists studying other virulent infections.

\section{RHEUMATIC PERICARDITIS.}

The consideration of the rheumatic lesions pericarditis, endocarditis, and myocarditis, apart from carditis, is necessarily artificial, but is essential on account of their individual importance.

Pericarditis is placed first here because of its gravity. It is in general terms a mark of a severe infection, and is repeatediy met with in fatal carditis. One hundred and fifty cases in children under 12 years of age showed at necropsy that the pericardium was adherent wholly or in part in 113 cases, and in only 9 cases was it stated to be healthy. In only 6 cases were more than $3 \mathrm{oz}$. of fluid recorded in the pericardial sac.

My experience both as a teacher and an examiner has shown me that the student looks upon rheumatic pericarditis as a disease in itself, running through three definite stages: (1) the stage of early inflammation; (2) of pericardial exudation; (3) of resolution.

In the general study of pericarditis such a conception is undoubtedly useful as a finger-post to guide us through the first difficulties of the subject, but it is not a correct or even satisfactory description of the rheumatic form in childhood.' It brings the element of effusion into too great prominence, both as an incident in the illness and as a factor in the production of grave symptoms, and places too much emphasis upon mechanical embarrassment of the heart, which in reality takes but little part in rheumatic pericarditis at this age. There seems by this view to be an idea that some mysterious stage of effusion must appear and disappear with the regularity almost of clockwork. But the pathological changes are much more complicated.

We know from experiment that rheumatic inflammation commences within the pericardial tissues and not upon the 
surfaces. In severe cases the foci are innumerable; in mild cases they are fewer and may not even damage the surface endothelium. The exudations vary, too, in character with the virulence and stage of the process. The worst cases show haemorrhagio exudations, but more usually they are fibrinoplastio or serofibrinous and seldom very copious. If the process is obstinate and subacute, the changes may be chiefly in the pericardial tissues, where nodular formations may occur and great thickening of the parietal layer follow. The amount of exudation in such a case will be small, if any, and the two diseased layers will become welded together by scar formation between the opposed surfaces. This condition is comparable to that of mitral stenosis.

The morbid process, commencing as it does in local foci, may severely implicate one part only of the pericardiumfor example, the posterior-and even when it is termed general there are often left comparatively untouched areas in which subsequently new lesions may arise causing pericardial friction, although at the necropsy the pericardium may appear to have been universally adherent.

In rare cases, again, a large effusion may give rise to special symptoms.

It is evident, then, that our conception of rheumatic pericarditis must be built up on a clinical study of individual cases, and we cannot assume a definite and regular sequence of events.

The practical application of these pathological changes in rheumatic pericarditis to clinical medicine is of great value. In this condition there is often great and some times extreme enlargement of the area of precordial dullness; and if we have trained our minds to a rigid adhesion to the three stages of pericarditis we naturally regard this increased area as the result, and further the measure, of effusion. Then naturally we shall regard the feebleness of the heart as the mechanical result of the effusion and be tempted to act upon this reasoning. What, however, is the real truth? It is this: In the vast majority of cases the increased area is the result of acute dilatation (added to, doubtless, in some degree by effusion) the dilatation itself being the result of a carditis. If wo rashly thrust a trocar into the area of increased dullness we should probably pierce the heart, as has been done on more than one occasion.

I am convinced that it is a very valuable aid to the physician (for it is no light undertaking to venture upon operative measures in pericarditis) if he realizes that however large the area of cardiac dullness in the rheumatic pericarditis of childhood it is exceptional for this to be the result of a large effusion.

There is another point which, while of lesser impor tance, must not be lost sight of. Pericarditis is rightly looked upon as a serious lesion, but we must beware of assuming that because pericarditis is not present the heart is not seriously damaged. Apart from the great difficulty in detecting some cases of pericarditis there may be occasions upon which it does not occur and ye the heart affection be very severe. These are examples of myocarditis which show the same dilatation and excited action as do the pericardial infections but they lack the clinical sign of friction. Such a heart may be far more seriously injured than one in which there has been general pericardial friction. I have seen, as have others, several cases of general pericarditis in strong children run rapid and most benign course, and later have failed to detect any fault but a slight mitral leak. This exemplifies a cardinal rule in the study of infective heart diseasethat we must be guided by the general results of the injury to the heart and to the patient, and not entirely by clinical terms such as pericarditis or a clinical sign such as pericardial friction.

These three points bear recapitulation :

1. The varying types of pericard is in rheumatic children.

2. The rarity of large effusions.

3. The dependence of the symptoms upon a concomitant corditis.

Pericarditis, in addition to the evidence it affords of severe infection, brings certain dangers of its own. Pain may be a prominent feature, increased no doubt in many cases by its association with pleurisy, and the supporting power of the pericardium is seriously injured when its tissues are severely inflamed. Doubtless the movements of the heart are embarrassed when the two surfaces of the pericardium are much roughened, and, though in childhood much effusion is rare, exceptions occur introducing these added difficulties. Very serious results will follow when the inflammatory process has spread through the outer surface of the parietal pericardium and invaded the mediastina, joining here with other inflammatory areas in the pleurae, for when healing occurs the matted tissues around the pericardium become welded to it and in turn to the heart by the internal pericardial adhesions. These complications are occurring at a time when the heart is dilated and the neuro-muscular tissues weakened by the disease. It is these particular results of pericarditis which make it necessary to consider the lesion apart from endocarditis and myocarditis, provided we also remember that these are usually present at the same time and add to the complication.

The chief clinical forms of pericarditis in childhood are :

1. Acute internal pericarditis with some effusion.

2. Acute pericarditis, internal and external, with mediastinitis and pleurisy.

3. Malignant pericarditis.

4. Relapsing cases.

These distinctions are to some extent artificial, but they are of value at the bedside because they illustrate the various courses that this lesion may take.

\section{Acute Pericarditis.}

Every textbook deals fully with the signs and symptoms of this lesion. As a rule the temperature is considerably raised, and there is a general illness with precordial pain, rapidity and feebleness of the pulse, and considerable dilatation.

Delirium appears to me to be very exceptional in childhood, and in some of the few cases I have met with chorea has seemed to me a factor in the mental state.

We rely greatly on pericardial friction for clinching the diagnosis, although we may be practically convinced by the general symptoms and trend of the case, even when friction has not been detected. Yet there are not in all cases of rheumatic pericarditis in childbood great distress and illness. On the contrary, there may be neither, and the physician may well be surprised when in a case of chorea with no apparent distress he discovers on examination of the heart very obvious pericardial friction. In fact, we can never safely forget in the rheumatism of childhood that the beart invariably requires careful physical examination.

Pericardial friction is a very constant sign in rhenmatic pericarditis, but I have not expressed this frequency in statistios because it is difficult to obtain accurate figures and they are open to considerable error. In general terms it may be stated that its occurrence is the rule and that the exceptions are either cases of active myocarditis or examples of pericarditis over a localized area on the posterior aspect, and in both instances the problems in treatment are closely akin.

When effusion occurs the cardiac sounds, as a rule, become less audible, and often the friction disappears or diminishes, but this is not constant, and it is well known that a loud pericardial rub may be heard even when there is a large effusion. More frequently the disappearance of the friction is followed shortly by its reappearance. The sound is then harsher and more grating, and is coincident with a general improvement in the child's condition. Clearly now the process of resolution is in progress, the two surfaces of the pericardium have approximated, and the exudation upon their surfaces has become more viscid.

The sequelae of pericarditis are beyond the scope of this article, but we have to remember that in the convalescence from acute pericarditis, provided there is no gross external adhesion, our attention must be shifted from the pericardium to the damaged myocardium.

-Endocarditis is almost invariable, though occasionally we find no evidence of its occurrence. This, however, is so unusual as to suggest that the pericarditis may be the result of some other infection-for example, the tubercular rather than the rheumatic-although I have convinced myself that this event may occur also in the rheumatic form. It does not follow that because endo. carditis is present we may at first be able to detect it, for the louder pericardial friction may entirely hide the softer valvular bruit. 
The course of an attack of acute pericarditis varies not only in virulence but with the resistance offered by the patient. There are exceptional cases in which the friction disappears in less than a week and the temperature reaching normal remains at this level. The dilatation rapidly subsides and the recovery may reach in three weeks the period of steady convalescence. Eventually recovery may be complete and even the mitral valve may have escaped involvement.

Far more frequently the active period is at least three woeks, during which time the friction may appear, dis. appear, and ugain become audible. There are pain and breathlessness with orthopnoea, a rapid pulse and irregular fever.

A close watch must be kept for increasing dilatation, progressive pallor, and increased frequency of the pulserate. Vomiting and restlessness are alarming symptoms warning us that the circulation is much enfeebled; a view confirmed by the appearance of slight enlargement of the liver, a scanty sometimes albuminous urine, and some oedema of the lungs. The physical signs over the chest posteriorly are often important. When there is great dilatation or an unusual degree of exudation, pressure upon the left lung and its bronchus will produce a condition of collapse of the lower lobe which may be mistaken for pneumonia or effusion. The note is impaired at the base behind and the breath sounds are intensely tubular at the level of the lower border of the scapula. No crepi tations need, however, be detected, and as the condition subsides the breath sounds become at first harsh and finally, in favourable cases, again normal.

It will greatly assist in the realization of this result on the left lung if an opportunity is obtained of seeing a necropsy upon a case of severe pericarditis with great cardiac dilatation. In such hardly any part of the lower lobe of the lung will be visible from the front, for it has been crowded back by pressure into the left axilla, and the condition of collapse will be very evident and the pathological signs produced easily understood.

I have only met with one case in childhood in which there has been an extreme effusion, producing embarrassment of the heart by its bulk and death by syncope. This was the case (No. 3) alluded to under acute carditis, and although operative mossures, as the event proved, would have been of no avail, measures, as the event proved, would have been of no avail, case was a mistaken one. In this instance there was fullness case was a mistaken one. In this instance there was fullness of the intercostal spaces on the left side over the precordial area, which should have led to a correct interpretation when
coupled with muffling of the cardiac sounds and the extreme distress.

A very remarkable coincidence followed this case in the admission of another child with severe pericarditis, muffling of the sounds, and an extreme area of cardiac dullness mounting as high as the second space on the right side. I was so unsteadied by my previous experience that I seriously considered surgical interference, but was checked by the very grave condition of the patient. The necropsy showed here that there was no fluid in the pericardium but sery mu thick a parietal pericardium with recent adhesion to an enormously dilated heart. I record this case here as a warning of the care that is neodod bolore deciding upon paracentesis of the periguidion. hasten to assume the presence of a large effusion.

\section{Acute Pericarditis, Internal and External, with} Mediastinitis and Pleurisy.

This group is a dangerous one, for it contains the cases in which the sequelae due to adhesions are the most serious. Here the pain and distress are greater, owing to the concomitant pleurisy. Pleuro-pericardial friction will be heard along the right and left margins of the cardiac dullness, and crepitations may be audible immediately under the stethoscope in the anterior mediastinum. In some cases the inflammation of the serous membrane is more extensive, and the surfaces of the liver and spleen are involved, and we hear the comparatively rare sign of peritoneal friction.

The course is a long one, extending, should the child survive the initial attack, over many weeks. The heart is greatly dilated, and remains so in spite of the greatest care in treatment. Later hypertrophy may develop, and then there may slowly supervene the signs of an adherent pericardium or of multiple serositis. To avoid any confu sion by use of the term external pericarditis, I mean by it inflammation of the outer surface of the parietal pericardiuinf:
Malignant Pericarditis.

This may give rise to some puzzling signs owing to the thickening of the parietal layer of the pericardium. In some cases at the necropsy this layer may be found a quarter of an incl in thickness, and will be loosely adherent to the visceral pericardium by recent adhesions.

The course is protracted, but when from the first the disease implicates the entire pericardium it runs a disastrous course, which is best exemplified by the following instance :

A boy aged 7 years had a severe attaok of carditis in the pre ceding year, and three weeks before his admission to hospital had sufered from multiple arthritis and precordiad pain. His fival illness lasted $8 \frac{1}{2}$ months, During this time there wer repeated nodules, and there was irregular fever reaching to $104^{\circ} \mathrm{F}$. From time to time pericardial friction appeared, and faded away only to reappear. He gradually became more anaemic and weaker, and fied of cardiao asystole. The necropsy showed great thickening of the pericardium and some recent adhesions; no fluid was present in the sac.

I have termed this a malignant rheumatic pericarditis because it suggests to me a process in the pericardium closely akin to that met with in the endocardium in malig. nant endocarditis. There is great local change from the micrococcal infection with the formation of nodular masses containing necrotic areas surrounded by greatly swollen and damaged connective tissue. There is also considerable persistent pyrexia. We must not, however, expect to meet with the widespread dissemination of foci of infection that occur in malignant endocarditis, for the pericardium does not bear the same relation to the general circulation as does the injured valve. Numerous infarctions, aneurysms, and such like phenomena will not occur, but the likeness lies in the obstinacy of the infection, the fever, and the severe general symptoms.

\section{Rerapsing Cases.}

In the relapsing form we see the link between the preceding and the more usual acute pericarditis. The sym. ptoms may never be severe, but the process advances by a series of local areas of infection, and pericardial friction may be audible at intervals for many weeks. When the rub disappears there is some degree of general improvement, when it reappears there is an exacerbation of the sym. ptoms. The pulse remains rapid and the heart is excited and dilated. Considerable thickening of the pericardium may result, and the heart itself is much damaged by chronic inflammation.

This coarse is likely to supervene in fragile, weakly patients who have little power of thoroughly destroying infection, and the outlook is gloomy although it may be difficult to realize the gradual sapping of the cardiac power while the child is in bed carefully nursed and guarded.

The thickening of the parietal pericardium, which occurs with the deposit of thick exudation upon the opposed surfaces coupled with the cardiac dilatation, may readily deceive those who are not acquainted with this form of pericarditis. The cardiac sounds are muffled and the area of precordial dullness is greatly enlarged, and may precisely resemble the outline that is met with when there is a large exudation. Further, the interpretation of an $x$-ray examination needs caution. The report may support the view of a large effusion based upon the outline of the shadow, and yet, as I have confirmed, the necropsy has proved the contrary to be the case.

If we can clearly discern the outline of the heart separated by a lighter area from the outline of the pericardium this interpretation of effusion is justified, but in these particular cases no such distinction between the two outlines will be found. An attempt in such a case to drain the pericardium can only lead to failure and possibly to disaster.

In these instances, in which the pericardium is so much swollen and injured by disease, there is a serious danger of the incomplete healing of the foci, and, with the persistence of lurking infection, of the development of the malignant form of pericarditis.

NoTE.-A kindly critic bas directed $m y$ attention to two badly expressed sentences in my first article. On p. 250 col. lines 33 and 34, for " small follicular deposits" read " col. deposits between the follicle"; line 49, "for internal ear " read 